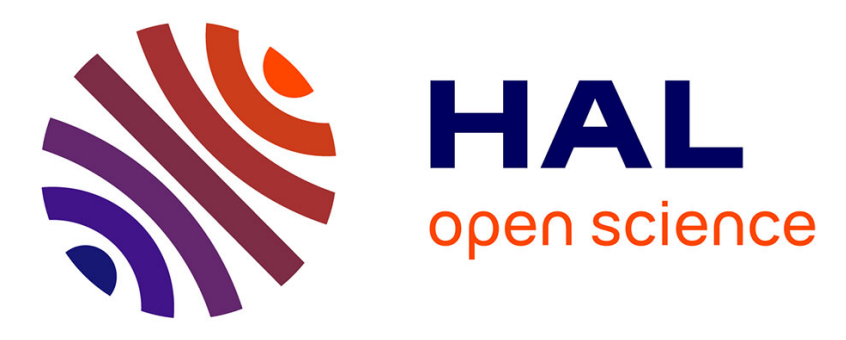

\title{
A Publish/Subscribe System Using Causal Broadcast Over Dynamically Built Spanning Trees
}

João Paulo de Araujo, Luciana Arantes, Elias P Duarte Júnior, Luiz A. Rodrigues, Pierre Sens

\section{To cite this version:}

João Paulo de Araujo, Luciana Arantes, Elias P Duarte Júnior, Luiz A. Rodrigues, Pierre Sens. A Publish/Subscribe System Using Causal Broadcast Over Dynamically Built Spanning Trees. SBAC-PAD 2017 - 29th International Symposium on Computer Architecture and High Performance Computing, Oct 2017, Campinas, Brazil. pp.161-168, 10.1109/SBAC-PAD.2017.28 . hal-01644469

\section{HAL Id: hal-01644469 \\ https://hal.inria.fr/hal-01644469}

Submitted on 22 Nov 2017

HAL is a multi-disciplinary open access archive for the deposit and dissemination of scientific research documents, whether they are published or not. The documents may come from teaching and research institutions in France or abroad, or from public or private research centers.
L'archive ouverte pluridisciplinaire HAL, est destinée au dépôt et à la diffusion de documents scientifiques de niveau recherche, publiés ou non, émanant des établissements d'enseignement et de recherche français ou étrangers, des laboratoires publics ou privés. 


\title{
A Publish/Subscribe System Using Causal Broadcast Over Dynamically Built Spanning Trees
}

\author{
João Paulo de Araujo*, Luciana Arantes ${ }^{\dagger}$, Elias P. Duarte Jr. ${ }^{\ddagger}$, Luiz A. Rodrigues ${ }^{\S}$ and Pierre Sens $₫$ \\ *† Sorbonne Universités, UPMC Univ Paris 06, CNRS, INRIA, LIP6 - Paris, France \\ ${ }_{\ddagger}^{\ddagger}$ Federal University of Paraná - Curitiba, Brazil \\ $\S$ Western Paraná State University - Cascavel, Brazil \\ Email: *joao.araujo@lip6.fr, †luciana.arantes@lip6.fr, †elias@inf.ufpr.br, §luiz.rodrigues@ ${ }^{\ddagger}$ unioeste.br, ๆpierre.sens@lip6.fr
}

\begin{abstract}
In this paper we present VCube-PS, a topic-based Publish/Subscribe system built on the top of a virtual hypercubelike topology. Membership information and published messages to subscribers (members) of a topic group are broadcast over dynamically built spanning trees rooted at the message's source. For a given topic, delivery of published messages respects causal order. Performance results of experiments conducted on the PeerSim simulator confirm the efficiency of VCube-PS in terms of scalability, latency, number, and size of messages when compared to a single rooted, not dynamically, tree built approach.
\end{abstract}

\section{INTRODUCTION}

Publish/Subscribe (Pub/Sub) systems consist of distributed nodes in which one or more publishers produce messages (events) that are consumed by subscribers. Communication between publishers and subscribers is conducted on an overlay infrastructure, which is generally composed by a set of nodes that organize themselves for ensuring the delivery of published messages to all (preferably only) interested subscribers. Therefore, publishers and subscribers exchange information asynchronously, without interacting directly [1], [2].

There basically exist two models of Pub/Sub systems: topicbased [3], [4], [5], [6] and content-based [7], [8]. In the first one, subscribers share a common knowledge on a set of available topics and every published message is labeled with one of these topics. A subscriber can register its interest in one or more topics, and then it receives all published messages related to these topics. In the content-based model, events are structured in multiple attributes, and subscribers express their interests by specifying constraints over the values of these attributes [9].

The advantage of the topic-based model is that events/messages can be statically grouped into topics, the diffusion of messages to subscribers is usually based on multicast groups, and the interface offered to the user is simple. Even if it offers limited expressiveness for subscribers [1], the topic-based approach is widely used by applications such as chat message systems, Twitter, mobile devices notification frameworks (e.g. Google Cloud Messaging), and many others. On the other hand, the content-based model provides more flexibility to subscribers for defining their event interests, but at the expense of more complex user interfaces and the need for filtering.
In this work, we are interested in topic-based Pub/Sub systems and, particularly, in offering an efficient support for publishers to send messages to subscribers, guaranteeing causal order among published messages, low latency, and load balancing.

In our proposed system, called VCube-PS, a user (node) can subscribe or unsubscribe to a topic $t$. After becoming a subscriber of $t$ and, therefore, member of the $t$ 's group, a node publishes a message $m$ associated to the topic $t$. Message $m$ is sent to all subscribers of $t$ using a broadcast protocol on top of a spanning tree, composed just by the subscribers, whose root is the publisher of $m$. This tree is dynamically built on top of a virtual hypercube-like topology, called VCube [10], that presents several logarithmic properties. Moreover, the tree construction itself has no overhead, since it is built using information nodes already have. We consider that nodes do not fail but can dynamically join to (make new subscription) or leave from (cancel subscription) one or more topic groups.

$V C u b e-P S$ also ensures causal ordering among published messages related to the same topic: if a node publishes a message after it delivers another message, then no node delivers the latter after the former. We note that in Pub/Sub systems the guarantee of published messages respecting causal order is an important and useful feature. For instance, in a discussion group, a question published on a group should never be delivered to any subscriber after an answer published in the same group, since question and answer are causally related. For sake of scalability, VCube-PS uses the causal barrier principle for implementing the causal order [11] where a message carries information about only those messages on which it directly depends.

Many Pub/Sub systems in the literature are based on broadcast trees [3], [4], [12]. However, they usually employ only one single generated tree, whose maintenance is sometimes costly when the membership of the system changes. Nodes which are not subscribers may take part in the tree as forwarders such as in Scribe Pub/Sub system [3], increasing the latency of message delivery, and the broadcast of a new publication is carried out by a single root node which, consequently, can become a bottleneck. In VCube-PS there is no single root node, as each node that publishes a message becomes the root of the corresponding spanning tree, providing load 
balancing. Furthermore, a spanning tree is composed only by the subscribers of the same topic and does not include forwarder nodes. A third point to emphasize is that very few $\mathrm{Pub} / \mathrm{Sub}$ systems in the literature (to the best of our knowledge, just JEDI [7]) provide causal ordering of published messages.

Experiments were conducted on the PeerSim simulator [13] and comparative results confirm the advantages of using perpublisher dynamically built spanning trees for load balancing, latency, number and size of messages, as well as the causal barrier approach for implementing causal ordering.

The rest of the paper is organized as follows. Section II introduces VCube. Section III presents the extensions and functions added to VCube in order to manage topics, order messages, and gives a description of VCube-PS's algorithms. Section IV presents evaluation results obtained from experiments conducted on PeerSim simulator. Section V discusses related work and, finally, Section VI concludes the paper.

\section{VCUBE}

In VCube [10], a node $i$ (also called $p_{i}$ ) groups the other $n-1$ nodes in $d=\log _{2} n$ clusters forming a $d$-VCube, each cluster $s(s>0)$ has size $2^{s-1}$. The ordered list of nodes in each cluster $s$ is denoted by function $c_{i, s}$ below, where $\oplus$ is the bitwise exclusive or operator (xor).

$$
c_{i, s}=i \oplus 2^{s-1} \| c_{i \oplus 2^{s-1}, k} \mid k=1, . ., s-1
$$

VCube is a distributed fault diagnosis system and it defines as the neighbors of a node $i$ the first faulty-free node of each cluster $s$. Periodically, $i$ tests the first node in the $c_{i, s}$ to check whether it is correct or faulty. Fig. 1 shows node $p_{0}$ 's hierarchical cluster-based logical organization of $n=8$ nodes connected by a 3-VCube topology as well as a table which contains the composition of all $c_{i, s}$ of the 3-VCube. Let's consider node $p_{0}$ and that there are no failures. The clusters of $p_{0}$ are shown in the same figure. Each cluster $c_{0,1}$, $c_{0,2}$, and $c_{0,3}$ is tested once, i.e., $p_{0}$ only performs tests on nodes $1,2,4$ which will then inform $p_{0}$ about the state of the other nodes of their respective cluster.

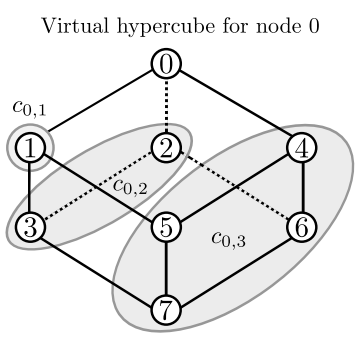

General $c_{i, s}$ table for 8 nodes

\begin{tabular}{|c|c|c|c|c|c|c|c|c|}
\hline$s$ & $c_{0, s}$ & $c_{1, s}$ & $c_{2, s}$ & $c_{3, s}$ & $c_{4, s}$ & $c_{5, s}$ & $c_{6, s}$ & $c_{7, s}$ \\
\hline 1 & 1 & 0 & 3 & 2 & 5 & 4 & 7 & 6 \\
\hline 2 & 23 & 32 & $\begin{array}{lll} & 1\end{array}$ & $\begin{array}{lll}1 & 0\end{array}$ & 67 & 766 & 45 & 54 \\
\hline 3 & 45567 & 5476 & 6745 & 7654 & \begin{tabular}{llll|l}
0 & 1 & 2 & 3
\end{tabular} & $\begin{array}{llll}1 & 0 & 3 & 2\end{array}$ & $\begin{array}{llll}2 & 3 & 0 & 1\end{array}$ & $\begin{array}{llll}3 & 2 & 1 & 0\end{array}$ \\
\hline
\end{tabular}

Fig. 1. VCube hierarchical organization.

\section{VCUBE-PS: PUbLISH/SUbSCRIBE SYSTEM}

We consider a distributed system composed of a finite set of $\Pi=\left\{p_{0}, . ., p_{n-1}\right\}$ nodes (users) with $n=2^{d}$ processes, $d>0$. Each node has a unique id and nodes communicate only by message passing. Each single node executes one task and a user of the Pub/Sub system corresponds to a node. Therefore, the terms node, user, and process are interchangeable in this work.

Nodes communicate by sending and receiving messages. The network is fully connected: each pair of nodes is connected by a bidirectional point-to-point channel and there is no network partitioning. Nodes do not fail and links are reliable. Thus, messages exchanged between any two processes are never lost, corrupted or duplicated. Each message is uniquely identified by the source id $(s)$, the topic $(t)$, and sequence number $(c)$ given by the logical clock of the source. The system is asynchronous, i.e., relative processor speeds and message transmission delays are unbounded.

$V C u b e$ has been extended to satisfy VCube-PS's needs. Thus, similarly to VCube, VCube-PS, organizes its nodes in a logical hypercube-like topology. However, as we consider that nodes do not fail, VCube-PS exploits VCube's organization but not its failure detection functionality. Even though it is possible to draw the analogy in which a node that has not subscribed to a topic $t$ is considered to be faulty in relation to $t$. Therefore, in VCube-PS, the first node of each cluster $s$ in $c_{i, s}$ in relation to topic $t$ should also be a subscriber of $t$.

The following functions are provided to node $i$ of VCube-PS by the virtual topology:

- $\mathrm{FF}_{-} \operatorname{NeIGHBOR}_{i}(t, s)$ : if $t={ }^{\prime} *$ ', no topic is considered and the function returns the first node in $c_{i, s}$; otherwise it returns the first node in $c_{i, s}$ which is also a member of group $t$. If there is no such a node, the function returns $\perp$ (no neighbor).

- Neighborhood $i(t, h)$ : returns a set that contains all nodes virtually connected to $i$ according to $\mathrm{FF}_{-} \operatorname{NeIGHBOR}_{i}(t, s)$, for $s=1, . ., h$ and topic $t$. The parameter $h$ can range from 1 to $\log _{2} n$. For $h=\log _{2} n$ the function returns all neighbors of $i$, if $t={ }^{\prime}$ ', no matter the topic; otherwise all neighbors of $i$ that are subscribers of $t$. For any other value of $h<\log _{2} n$, the function returns only a subset of the first neighbors, i.e., those first neighbors whose respective cluster number $s$ is smaller or equal to $h$ $(s \leq h)$. For instance, in Fig. 1, if $t={ }^{*} *$ ', NeIGHBor$\operatorname{HOOD}_{0}(*, 3)=\{1,2,4\}, \operatorname{NeIGHBORHOOD}(*, 2)=\{1,2\}$, and NeIGHBORHOOD $4(*, 2)=\{5,6\}$. On the other hand, if only nodes 0,3 , and 4 have joined topic $t_{1}$, NEIGHBOR$\operatorname{HOOD}_{0}\left(t_{1}, 3\right)=\{3,4\}$ and NeIGHBORHOOD $4\left(t_{1}, 2\right)=\perp$.

- $\operatorname{Cluster}_{i}(j)$ : The function returns the index $s$ of the cluster of node $i$ that contains node $j,\left(1 \leq s \leq \log _{2} n\right)$. For instance, in Fig. 1, $\operatorname{CLuster}_{0}(1)=1, \operatorname{CLuster}_{0}(2)=$ $\operatorname{Cluster}_{0}(3)=2$, and Cluster $(4)=\operatorname{CLuster}_{0}(5)=$ $\operatorname{CLuster}_{0}(6)=\operatorname{CLuster}_{0}(7)=3$.

Causal and Per-source FIFO Reception Orders: For each topic, VCube-PS respects causal order of published messages, 
implementing, thus, a causal broadcast.

Causal Order: if a process publishes a message $m^{\prime}$ after it has delivered another message $m$, then no process in the system will deliver $m$ after $m^{\prime}$.

Note that if a process $i$ never delivers $m^{\prime}$ (e.g., $i$ leaves the system before delivering $m^{\prime}$ ) or delivers $m^{\prime}$ but never delivers $m$ (e.g., $i$ was not in the system when $m$ was published), the causal order of published messages is not violated.

In order to implement the causal order of published messages, we apply causal barriers [11]. The advantage of the causal barrier approach is that it does not control causality based on nodes' identifiers (per node vector) but by using direct dependencies of messages which renders the algorithm more scalable and suitable for dealing with the dynamics of nodes (subscriptions and unsubscriptions).

Let $m$ and $m^{\prime}$ be two application messages published for topic $t$. Message $m$ immediately precedes message $m^{\prime}$ (denoted $m \prec_{i m} m^{\prime}$ ) if (1) the publishing of $m$ causally precedes the publishing of $m^{\prime}$ and (2) there exists no message $m^{\prime \prime}$ such that the publishing of $m$ causally precedes the publishing of $m^{\prime \prime}$, and the publishing of $m^{\prime \prime}$ causally precedes the publishing of $m^{\prime}$. The causal barrier of $m\left(c b_{m}\right)$ consists of the set of messages that are immediate predecessors of $m$.

Fig. 2 shows a distributed system with three nodes $\left(p_{0}, p_{1}\right.$, and $p_{2}$ ) that have subscribed to the same topic $t$. Message $m_{s, t, c}$ is the message published by $s$ with sequence number $c$ for topic $t$. On the left side of the figure, we have a timing diagram with the publishing and delivery of messages and on the right the graph with message dependencies. We can observe that the delivery of $m_{1, t, 1}$ is conditioned by the delivery of $m_{0, t, 1}\left(m_{0, t, 1} \prec_{i m} m_{1, t, 1}\right)$ since $p_{1}$ delivered $m_{0, t, 1}$ before publishing $m_{1, t, 1}$, (i.e., $c b_{m_{1, t, 1}}=\left\{m_{0, t, 1}\right\}$ ). On the other hand, $m_{1, t, 2}$ directly depends on $m_{2, t, 1}$ and $m_{1, t, 1}$ (i.e., $c b_{m_{1, t, 2}}=\left\{m_{2, t, 1}, m_{1, t, 1}\right\}$ ). Note that since $m_{0, t, 1}$ precedes $m_{1, t, 1}$ that precedes $m_{1, t, 2}, m_{0, t, 1}$ is an indirect dependency of $m_{1, t, 2}$, not included, therefore, in $c b_{m_{1, t, 2}}$.

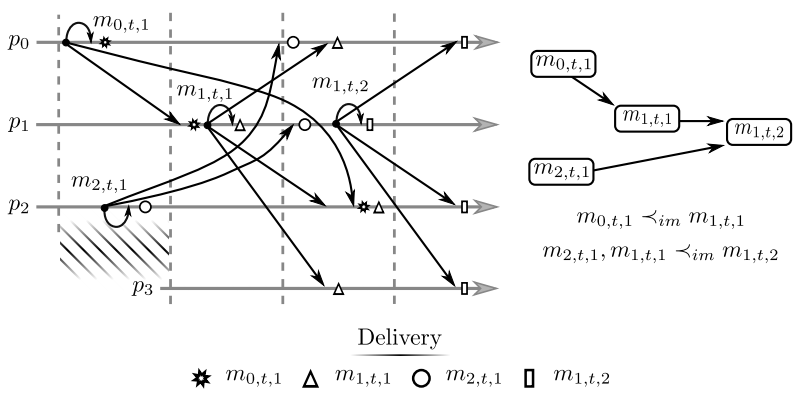

Fig. 2. Example of causal barrier.

Let's now suppose, in the same figure, that $p_{3}$ subscribes to $t$ after message $m_{2, t, 1}$ was published to the other nodes, i.e., in this case, node $p_{3}$ will never receive nor deliver $m_{2, t, 1}$. Hence, after having delivered $m_{1, t, 1}, p_{3}$ can deliver $m_{1, t, 2}$.

Since nodes can dynamically subscribe to or unsubscribe from a topic in $V C u b e-P S$, our implementation of message causal order must distinguish between the case in which a message will be delivered (e.g., $m_{1, t, 1}$ ) from the one that it will never be delivered (e.g., $m_{2, t, 1}$ by $p_{3}$ ). To this end, VCube$P S$ guarantees the following property on the FIFO order of messages published on a given topic.

Per-source FIFO Reception Order: messages published by a same publisher are received by subscribers in the same order as they were produced. In other words, $m_{s, t, c}$ can be broadcast to the current subscribers of $t$ provided that the previous message $m_{s, t, c-1}^{\prime}$ was received (not necessarily delivered) by all the subscribers present in the system when $m^{\prime}$ was broadcast. This order allows a subscriber of $t$ to know that it will never receive some messages previously published, i.e., if $m_{s, t, c^{\prime}}^{\prime}$ is the first message that $i$ receives from $s$ on topic $t$ after it joined $t$ 's group, $i$ will never receive $m_{s, t, c}$, if $c<c^{\prime}$.

In VCube-PS, per-source FIFO reception order is ensured by the acknowledgment of published messages: a source node broadcasts a new message only after having received all the acknowledgments for the previous message it had previously broadcast. Note that the per-source FIFO reception order is defined in regard to the reception of messages and not delivery, as in the traditional FIFO order definition.

VCube-PS Algorithm Description: Due to lack of space, we do not present the algorithms that manage topic membership and disseminate published messages to subscribers of a given topic. The latter can be found in Section III of [14]. In the following, we give a description of the algorithms.

Application Functions: VCube-PS offers to the application the functions $\operatorname{Subscribe}(t), \operatorname{Unsubscribe}(t)$, and $\operatorname{PUBLISH}(t, m)$ which allow a user (node) to subscribe to topic $t$, unsubscribe from $t$, and publish a message to all subscribers of $t$, respectively. A node can publish a message related to a topic provided it is currently a subscriber of this topic. Those functions will generate messages of type SUB, UNS, and PUB respectively which will be sent to all nodes, in case of subscription, or to all subscribers of $t$, otherwise. Every message is uniquely identified by the source id, the topic, and a sequence number (counter) in regard to this topic. In the case of a message of type PUB, it also carries the identifiers of direct causally related messages (causal barrier). After a function is invoked, a distributed spanning tree dynamically built and rooted at the caller is used to disseminate the corresponding messages.

Producer/consumer approach: Since in Pub/sub systems the publishing of a message must be decoupled from its delivery by the subscribers, in VCube-PS, every message generated by the execution of a function by $i$ is inserted in a queue per topic $t$. Then, a per topic task at $i$ continuously removes the first message $m$ from this queue and starts its broadcast. The next message is removed from the queue only when $i$ is sure of the reception of $m$ by all current subscribers (per-source FIFO reception order), i.e., reception of acknowledgement (message of type ACK) from those nodes to whom $i$ sent $m$.

Propagation of a message: For every message $m$ taken from the queue of node $i$ associated to topic $t$, the corresponding task starts the propagation of $m$ by dynamically building a 
hierarchical spanning tree, rooted at $i$, composed by the nodes which are either subscribers of $t$, in case of messages of type UNS or PUB, or composed by all nodes, in case of messages of type SUB. For this purpose, $i$ calls function $\operatorname{NEIGHBORHOOD}_{i}(t, h)$ which renders the set of the first subscribers of $t$ for each of the $\log _{2} n$ clusters of process $i$ (in the case of SUB messages, $t=$ ' $*$ '). These neighbors become $i$ 's children in the spanning tree and $m$ is sent to them. Upon the reception of $m$, by calling function $\operatorname{CLUSTER}_{j}(i)$, every $i$ 's child $j$ sends $m$ to its $s-1$ first neighbors of $j$ in relation to topic $t$ and the cluster $s$ of $i$ to which $j$ belongs, i.e., $c_{i, s}$. These neighbors then become $j$ 's children, and the process continues as described in the following example.

For instance, consider in Fig. 1 that either node $p_{0}$ is not a subscriber to $t_{1}$ and wants to subscribe or it is already a subscriber and wants to publish a message related to $t_{1}$. All other nodes are already subscribers. Node $p_{0}$ sends a message $m$ related to $t_{1}$ (SUB or $\mathrm{PUB}$ ), becoming then the root of the respective spanning tree. The message will be sent to the $\log _{2} n=3$ children of $p_{0}: \mathrm{FF}_{-} \operatorname{NEIGHBOR}_{0}\left(t_{1}, 1\right)=1$, $\mathrm{FF}_{-} \mathrm{NEIGHBOR}_{0}\left(t_{1}, 2\right)=2$, and FF_NEIGHBOR $0\left(t_{1}, 3\right)=4$. Upon the reception of $m, p_{1}$ does not forward it since its $\operatorname{CLuster}_{1}(0)=1$, while $p_{2}\left(\operatorname{CLuster}_{2}(0)=2\right)$ forwards it to its child $p_{3}$, the first subscriber of its cluster $1\left(c_{2,1}\right)$. When $p_{3}$ receives $m$, as its $\operatorname{CLUSTER}_{3}(2)=1, p_{3}$ does not forward $m$ to any node. However, in the case of $p_{4} \operatorname{(CLUSTER}_{4}(0)$ $=3$ ), it forwards $m$ to its children $p_{5} \in c_{4,1}$ and $p_{6} \in c_{4,2}$. Finally, since CLUSTER 6 (4) $=2, p_{6}$ sends $m$ to $p_{7}$.

Let's consider now a second example also using the same Fig. 1 where only $p_{0}, p_{2}, p_{4}, p_{5}$, and $p_{6}$ nodes are subscribers of $t_{2}$ and $p_{2}$ publishes $m^{\prime}$ related to $t_{2}$. In this case, $p_{2}$ sends $m^{\prime}$ to the first neighbors of its $\log _{2} n=3$ clusters that are also subscribers of $t_{2}$ : FF_NEIGHBOR $2\left(t_{2}, 1\right)=\perp$ (there is no subscriber $t_{2}$ in cluster $\left.c_{2,1}\right)$, FF_NEIGHBOR $2\left(t_{2}, 2\right)=0$, and $\mathrm{FF}_{-} \mathrm{NEIGHBOR}_{2}\left(t_{2}, 3\right)=6\left(p_{6}\right.$ is the first subscriber $t_{2}$ in $\left.c_{2,3}\right)$. Upon receiving $m^{\prime}, p_{0}$ does not forward it to $p_{1}$ since the latter is not a subscriber of $t_{2}$. On the other hand, $p_{6}$ verifies that in cluster $c_{6,2}=(4,5), p_{4}$ is also a subscriber of $t_{2}$, and therefore sends $m^{\prime}$ to $p_{4}$ which then sends $m^{\prime}$ to $p_{5}$, the first and only $t_{2}$ 's subscriber of cluster $c_{4,1}$.

After forwarding a message $m$ to a child $j$, node $i$ waits for an ACK message from $j$, which confirms the reception of $m$ by $j$. However, $j$ will only send an ACK back to $i$ after receiving itself $\mathrm{ACK}$ messages from all its current children. ACK messages will, thus, be propagated from the leaves to the root, the source node of $m$. Eventually, the latter receives all the ACK messages from its children and, in this case, the task related to $t$ removes the next message to be published from the queue of the topic $t$, if one exists. These sequences of SUB, UNS, or PUB and then ACK messages from/to the source ensure the per-source FIFO reception order of published messages of a topic. In the case of SUB messages, the ACK messages will gather information about the membership of $t$ and, therefore, $i$ becomes aware of current subscribers.

Reception and delivery of messages: When receiving a $\mathrm{PUB}$ message $m$ of topic $t$ from $s$, if $i$ is a subscriber of $t$ and has not already delivered $m, i$ keeps $m$ and then verifies, based on direct causal dependencies, which received messages can be delivered to the application. A message $m$ can be delivered to $i$ only when every message $m^{\prime}$ on which $m$ causally depends has been either already delivered by $i$ or will never be received by $i$ since $V C u b e-P S$ has not considered $i$ as a subscriber of $t$ during the construction of the spanning tree which broadcast $m^{\prime}$. This determination of the first message is possible thanks to the fact that, for the same source, the publication of messages of the same topic respect per-source FIFO order. In the case of a SUB (resp., UNS) message, $i$ includes (resp., removes) the identity of the source of the message in (resp., from) its $t$ group membership.

\section{EXPERIMENTAL RESULTS}

In order to assess the performance of $V C u b e-P S$ with different configuration scenarios, we conducted experiments on top of the event-driven PeerSim [13] simulator. In the majority of scenarios, we compared VCube-PS with an approach denoted SRPT (Single Root Per Topic), which is similar to Scribe [3], where a single multicast tree per topic, composed by both subscribers and non-subscribers (forwarders) is used to publish all messages of a topic. SRPT maps each topic into a node that acts as the root of the broadcast tree for the respective topic. Forwarders receive and re-transmit published messages but do not deliver them.

In the experiments, we consider that each message exchanged between two processes consumes $t_{p c}+t_{q}+t_{t}+t_{p p}+t_{d}$ units of time (u.t.). Apart from $t_{d}$ which represents the time necessary for a subscriber to satisfy all causal dependencies before delivering a message to the application, all other components are based on a packet-switched network delay model [15]: $t_{p c}$ accounts for the processing time of a message by a node, e.g., checksum verification and routing decisions; $t_{q}$ is the time a message must wait in the queue before being transmitted; $t_{t}$ is the time necessary to transmit all bits of the message into the link, and $t_{p p}$ expresses how long it takes for a message to transverse the link and reach the next hop. Assuming that there is no broadcast facility available in the system, if a message is sent to multiple destinations, a copy of it is queued for each of the destinations. For our experiments, the ratio between $t_{p c}$ and $t_{p p}$ has an impact on the threshold value for starting to queue messages as well as how fast the queue grows. Hence, based on [16], we set $t_{p c}=t_{t}=1$ u.t. and $t_{p p}=100$ u.t. (1/100 ratio). The time a message stays queued $\left(t_{q}\right)$ is a function of the rate of incoming/outgoing messages and can vary for each message. Likewise, when a node $i$ receives a message $m, t_{d}$ will depend on how fast $i$ will receive all the preceding messages of $m$. If there is no preceding message $t_{d}=0$.

The number of nodes $n$ used in the simulations varies from 8 up to 1024 , in a power of two. Each experiment was executed 40 times and for the results that comprise average values we also provide the standard deviation.

We consider the following metrics: 
- Latency: the time that a published message takes to be delivered by all subscribers.

- Number of messages: overall number of PUB messages.

- Number of messages to be processed by a node: size of the queue of each node.

- Size of PUB messages: characterizes the number of direct causal dependencies that PUB messages hold.

- Number of false positives: number of messages received by nodes that act as forwarders of messages of type PUB.

Scenario with one publisher: The aim of this experiment is to evaluate the impact of the logarithmic properties of $V C u b e-P S$. We consider that one publisher broadcasts only one message. Hence, when a subscriber receives the message, there is no delay for delivering it, since causal order of published messages is not applied in this case. Fig. 3 depicts the delivery latency when the number of nodes of the system varies and either $100 \%$ or $25 \%$ of them are subscribers. The set of subscribers is randomly chosen following a uniform distribution and fixed at the beginning of each execution. We should remark that when $100 \%$ of the nodes are subscribers, $S R P T$ has no forwarder and, therefore, the latency for both $\mathrm{Pub} / \mathrm{Sub}$ systems is always proportional to $\log _{2} N$. The only difference in this case is that SRPT has an additional hop as the message to be published must be sent to the root of the single tree.

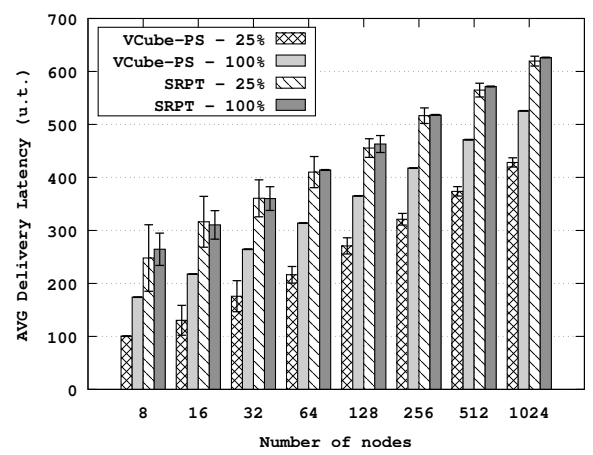

Fig. 3. Average latency for the delivery of one message to each subscriber.

In the case of 1024 nodes with $25 \%$ of subscribers uniformly distributed, the latency in VCube-PS is in average 420 units of time which results in a reduction of $31 \%$ compared to the latency presented by SRPT in the same scenario (620 u.t.) The higher standard deviation observed for SRPT with $25 \%$ of subscribers in scenarios with fewer nodes is due to the greater number of forwarders that have an impact in the average of the delivery time of all nodes. On the other hand, as the number of nodes increases, the number of samples (delivery time at each node) tends to a similar value as we average all executions. Moreover, with $100 \%$ of subscribers in SRPT, the standard deviation may be higher than zero since there exists a probability that the root node is the publisher itself and, therefore, SRPT performs just like VCube-PS, with no additional hop.

Forwarders induce an increase in the number of messages propagated throughout the network. Fig. 4 depicts the average

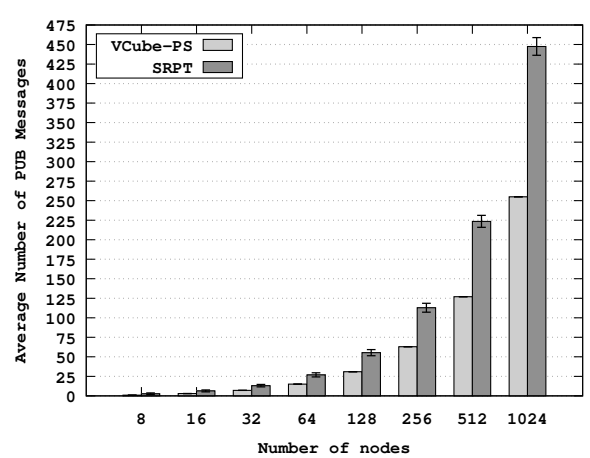

Fig. 4. Average number of PUB messages with $25 \%$ of subscribers.

number of PUB messages for the two approaches with $25 \%$ of the nodes as subscribers. In VCube-PS, this number is always equal to the number of subscribers, since there is no forwarder in the multicast tree. On the other hand, the forwarders in $S R P T$ are responsible for up to 2.7 times more messages (for 8 nodes) when compared to VCube-PS. As the number of nodes increases, this difference is reduced, although VCube$P S$ generates, in average, at least $43 \%$ fewer messages than SRPT (1024 nodes).

Scenario with several publishers: In these experiments, all nodes are subscribers of a single topic and the number of publishers varies. Each publisher $i$ sends one message at time $t_{i}$ which is chosen by a uniform distribution between $[0,1000]$ units of time. Fig. 5 shows in logarithmic scale the average reception latency when the number of nodes of the system varies and either $100 \%$ or $25 \%$ of them are publishers. Since the ratio between the processing time $\left(t_{p c}\right)$ andthe propagation time $\left(t_{p p}\right)$ has an impact on the load contention, we consider the ratio $1 / 100$ (Fig. 5(a)), which is used in all other evaluation of this work, but also a propagation time which is ten times greater, $\left(t_{p p}=1000\right.$ u.t.), leading to a ratio $1 / 1000$ (Fig. 5(b)).

We can observe in Fig. 5(a) the stability of VCube-PS for both percentages of publishers, with a maximum increase of $5.7 \%$ (1024 nodes and $100 \%$ of publishers). This result corroborates that the use of one tree per publisher helps to distribute the load, since each message traverses a different path in the network. On the other hand, as SRPT imposes a unique tree for disseminating messages to subscribers of a topic, if several messages arrive at the root node of the tree at the same time they will be queued before transmission, increasing, thus, the reception latency. The numbers for SRPT up to 128 nodes are in average one hop in time higher compared to VCube-PS due to the additional hop used by $S R P T$. The arrival rate of messages in this case is close to the output rate, leading to no contention. Beyond this number of nodes, the root receives more messages than it can process and transmit per interval of time and starts to saturate.

Comparing Fig. 5(b) with Fig. 5(a), we observe a lower increase in the average reception latency for $S R P T$ in relation to VCube-PS since a $1 / 1000$ ratio induces a higher latency in the reception of messages by a node, although its output throughput remains the same. Fig. 5(b) shows an increase of 


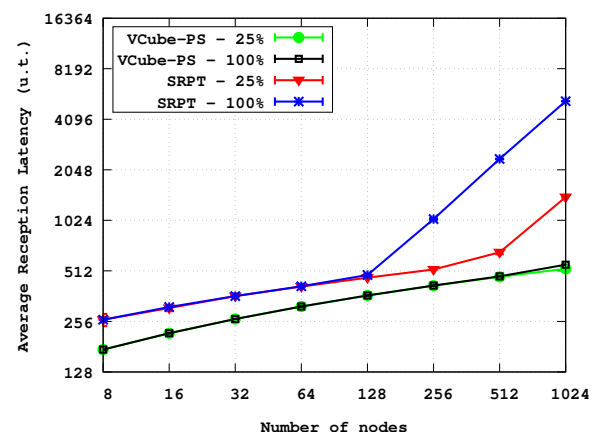

(a) $1 / 100$ ratio $\left(t_{p p}=100\right)$.

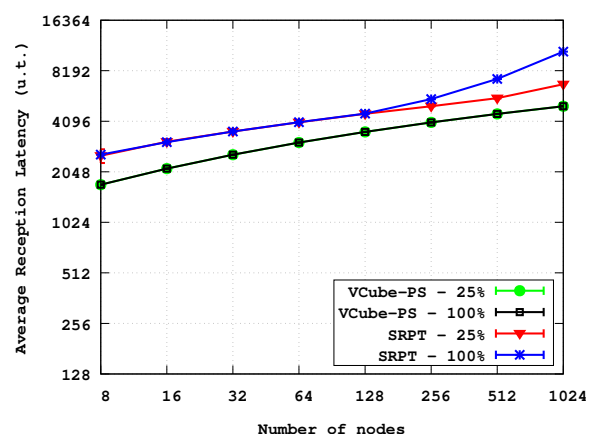

(b) $1 / 1000$ ratio $\left(t_{p p}=1000\right)$.

Fig. 5. Average reception latency with $25 \%$ and $100 \%$ of publishers (logarithmic scale).

TABLE I

AVERAGE SIZE OF THE QUEUE PER GROUP OF NODES.

\begin{tabular}{|c|c|c|}
\hline \hline \# of messages & \# of nodes (VCube-PS) & \# of nodes (SRPT) \\
\hline 0 & 0 & 512 \\
\hline$(0,2]$ & 0 & 448 \\
\hline$(2,4]$ & 0 & 60 \\
\hline$(4,8]$ & 495 & 3 \\
\hline$(8,16]$ & 510 & 0 \\
\hline$(16,32]$ & 19 & 0 \\
\hline$(32,4096]$ & 0 & 0 \\
\hline$(4096,8192]$ & 0 & 1 \\
\hline \hline
\end{tabular}

only $37 \%$ for 256 nodes ( $100 \%$ of publishers).

Table I shows the distribution of messages among nodes. For 1024 nodes and a ratio of $1 / 100$ where all nodes are publishers and subscribers, the table groups nodes with similar average size of queue (given by intervals).

We observe an uneven distribution of the load among the nodes in SRPT when compared to VCube-PS: $98 \%$ of the nodes in VCube-PS have an average load between $(4,16]$ messages while, although $44 \%$ of the nodes in SRPT have in average between $(0,2]$ messages in their buffers, $50 \%$ of the nodes simply do not participate of the routing of any message. Moreover, in SRPT, one node (the root of the tree) has an average load of $9240(\sigma=4617)$ messages, which is a bottleneck that increases the overall reception latency and limits the load of the other nodes to just a few messages. Besides that, since each node publishes one message, in VCube-PS we have 1024 different trees which leads to an uniform distribution of sent messages where any node transmits exactly 1024 messages. On the other hand, in SRPT the relationship between the number of nodes and the amount of messages they publish is reduced exponentially.

Ordering of messages: In order to evaluate the size of messages and the latency due to message ordering, we consider that one node $s$, chosen randomly, publishes a first message $m_{s}$. Upon receiving it, each node $k$ waits for a random time $\left(t_{w}\right)$ before broadcasting message $m_{k}$, similarly to a message discussion group service. Additionally, a node $k$ has to wait at least $p$ messages before broadcasting its own. To this end, there are $j \geq 1$ nodes that independently broadcast a message each in the beginning of the experiment. Just after receiving all these initial messages, any node can publish a message.

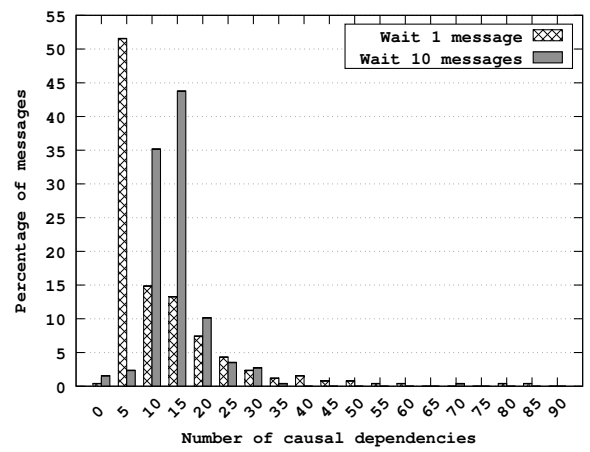

Fig. 6. Frequency distribution for the number of causal dependencies of a message in a network running VCube-PS with 256 nodes.

Fig. 6 groups messages according to the size interval of their causal barriers for VCube-PS. When it is necessary to wait just one message before a node broadcasts its own message, $51.6 \%$ of the messages generated in the system have less than 5 preceding messages. More precisely, $19.9 \%$ of all messages have just one causal dependency. On the other hand, if a node has to wait for more messages, 10 in the case of Fig. 6, before broadcasting its own, a larger number of nodes will have 10 or more direct dependencies. In this case, $35.2 \%$ of the messages have size 10 (10 direct dependencies) and $79.7 \%$ of them have fewer than 15. However, in both cases, due to both the use of causal barriers and multiple tree-roots used by VCube-PS, the size of the dependency list of most of the messages keeps close to the dependency threshold.

It is also worth to evaluate the additional delay imposed by the causal barrier before delivering a message to the application. If messages take too long to be delivered, this delay may lead to a cascade effect that could make the system unfeasible. In our simulations, when a node waits for 1 message before broadcasting its own, about $95.1 \%$ of the messages are delivered in less than 10 u.t. after the message is received $(87.2 \%$ are delivered with no delay). Only 81 messages (out of 65280) have a delay higher than 50 u.t., with an upper limit of 150 units of time. Increasing the number of the waiting messages to 10,457 messages wait 
more than 50 u.t. to be delivered (maximum 187), although the number of messages with no delay remains high $(84.2 \%)$.

Scenario with several topics: In order to evaluate the popularity of topics in Twitter, the authors in [17] carried out some experiments that show that roughly $60 \%$ of the topics have only one message published and $83 \%$ of them have no more than 5 . On the other hand, only $0.15 \%$ of the topics are related to more than 1000 messages each. This behavior follows a Zipf-like distribution with a coefficient of 0.825 according to the data provided in the article.

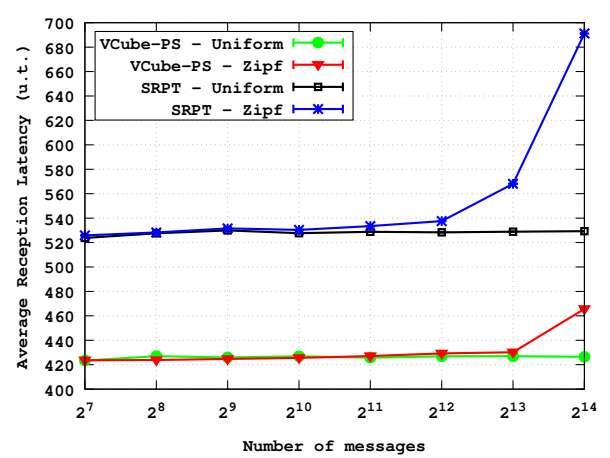

Fig. 7. Average reception latency with 256 nodes and 128 topics for two distribution of messages per topic.

Fig. 7 presents the average reception latency for 256 nodes and 128 topics where the number of published messages varies. The topic associated with each message is chosen following one of the two distributions: uniform or Zipf with a coefficient equals to 0.825 . Each node publishes a new message in average every 500 u.t. for a topic randomly chosen until a maximum number of messages is reached. Therefore, the messages are uniformly distributed among the publishers, but not necessarily among the topics. The minimum difference of around 100 u.t. is due to the additional hop used by SRPT to send a message to the root of the topic. No matter the distribution of messages among the topics, VCube-PS always relies on the same root for a given node, while SRPT may take advantage in the case of a uniform distribution. This is the reason why the behavior of SRPT is the same as VCube$P S$ 's for a uniform distribution of messages. However, when the number of messages sent per node increases beyond a threshold, VCube-PS increases latency due to contention at the source of the messages, i.e., the root of the tree. On the other hand, for the Zipf distribution, SRPT has an average reception latency $30.6 \%$ higher compared to the uniform distribution (for $2^{14}$ messages). For this same situation, VCube-PS increases the latency, in average, only $9.2 \%$. In a non-uniform distribution, the higher the number of messages, the closer the behavior becomes to that discussed previously with several publishers.

The use of a tree rooted on the a message's source makes $V C u b e-P S$ scalable in terms of publishers, while SRPT is scalable in terms of topics. However, in a real scenario, like the one presented by [17], even if a large number of topics may exist, most of the messages are concentrated on a small number of topics.

Scenario with dynamics of subscriptions: In these experiments, we are interested in evaluating how VCube-PS adapts itself to changes in the membership set of a topic.

When a node $i$ leaves a given topic $t$, it can still receive some messages addressed to $t$. It happens because not all other subscribers of $t$ received $i^{\prime} s$ unsubscription message yet. We classify these messages as false-positives. However, this is a temporary situation, since after a finite time interval other subscribers will receive the unsubscription message from $i$. In order to assess this behavior, we consider one publisher that publishes several messages on topic $t$. Due to the Persource FIFO property, one message will be broadcast after the reception of the ACKs from the previous one. For a given number of nodes, $75 \%$ of the nodes are randomly chosen as subscribers of a topic. Furthermore, at the same time the publisher starts the broadcast of its first message, $12.5 \%$ or $25 \%$ of the subscribers leave and new ones join the topic. The publisher sends 256 messages. Table II summarizes the results for this scenario.

TABLE II

DYNAMICS OF SUBSCRIPTIONS.

\begin{tabular}{|c|c|c|c|}
\hline \hline Churn & Nodes & AVG false-positives (std dev) & AVG deliveries (std dev) \\
\hline $12.5 \%$ & 512 & $2433.1(764.4)$ & $97445.5(655.7)$ \\
\hline $12.5 \%$ & 1024 & $10525.7(1207.1)$ & $194506.8(943.6)$ \\
\hline $25 \%$ & 512 & $3475.9(899.9)$ & $96193.9(1001.7)$ \\
\hline $25 \%$ & 1024 & $14590.4(1717.8)$ & $191706.3(1549.8)$ \\
\hline
\end{tabular}

The number of false-positives reaches at most $7.6 \%$ of the total number of delivered messages (1024 nodes with $25 \%$ of churn). In this case, 192 nodes leave the topic at the same time. In the best case, all other subscribers are notified in up to $\left(\log _{2} n * t_{p p}\right)$, but it is necessary to consider the queuing of messages in other nodes of the spanning tree (including unsubscriptions and new subscriptions). With 1024 nodes and $75 \%$ of them as subscribers, 196608 messages should be delivered. However, when there is $25 \%$ of churn, in average $97.5 \%$ of these messages are delivered.

\section{RELATED WORK}

Numerous Pub/Sub systems in the literature, such as Scribe [3], Bayeux [4], DYNATOPS [6], and Dynamoth [5], are based on topics. Compared to content-based, topic-based systems provide simpler and more efficient implementations. They are usually deployed in contexts where efficient and fast notifications are required.

Similarly to VCube-PS, many Pub/Sub systems use treebased overlays (e.g., Scribe [3], Bayeux [4], Marshmallow [12], DR-Tree [8], DYNATOPS [6]). The advantage of using trees is the logarithmic guaranties on publication delivery time and the number of messages employed. However, different from VCube-PS, most solutions often employ one single multicast tree (usually one per topic in topic-based systems), statically constructed from the start or as nodes join the system. Consequently, every publication should be 
broadcast from the root of this tree that might, then, become a bottleneck. Moreover, many of these multicast trees include unrelated intermediate hops and nodes that are not subscribers which have to forward the message, presenting thus the problem of false positives and the need of message filtering (e.g. DR-Tree [8], Scribe [3]). Finally, their maintenance cost is usually high, specially in presence of churn.

Several solutions (e.g. Scribe [3], DYNATOPS [6], etc.) construct independent multicast trees on top of Distributed Hash Table (DHT) overlays (e.g Pastry, CAN). They adopt the rendezvous point approach, where a node, responsible for the hashed key of a topic name, becomes the rendezvous point, i.e., the root of the multicast related to the topic. Some DHT overlay like PeerCube [18] and HOMED [19] are based on a hypercube-like topology themselves.

Even if defining a coherent order for notifications in $\mathrm{Pub} / \mathrm{Sub}$ systems is fundamental, few of them support event ordering [20], [7], [21], [22], specially total order. The authors in [20] propose a top-basic Pub/Sub system where messages published on different topics are either delivered in the same order to all subscribers or tagged as out-of-order (weak total order); while in [21], the task of ordering messages is distributed across sequencer nodes which totally order messages for the same topic. Considering FIFO links, Zhang et al. present in [22] a distributed total order protocol for a contentbased Pub/Sub system where a broker can determine if a message can be delivered immediately or, by collaborating with other brokers, that a consistent delivery order is required. JEDI [7] is a Pub/Sub system that ensures causal order. The latter is implemented by the use of a return value, a message by which a receiver notifies an event delivery to the producer of the event, unlike VCube-PS, which does not require these extra messages since the causal dependencies of a message are included in the message itself (causal barriers).

\section{CONCLUSION}

This work presented VCube-PS, a distributed topic-based $\mathrm{Pub} / \mathrm{Sub}$ system. Spanning trees are dynamically built on the top of a virtual hypercube-like topology and are used to both propagate information about membership changes and disseminate published messages to subscribers. While other approaches use a node as the rendezvous point of the tree of a topic, we configure a distributed spanning tree rooted on the source of every message, without any extra cost due to $V C u b e$. The trees contain only subscribers of the topic which induce trees of shorter height when compared to a per-topic single root tree and, therefore, lower latencies and smaller number of messages. VCube-PS also provides causal ordering of messages whose implementation uses the causal barrier approach, adapted to cope with the dynamics of the system.

Experimental results on PeerSim confirm the logarithmic behavior of VCube-PS. Compared to an approach with one single root per topic, our solution performs better when there is a high publication rate per topic since it provides load balancing of publication. Furthermore, VCube-PS does not present permanent forwarders which induce false positives, but, due to subscription dynamics, only temporary ones which eventually do not take part in any spanning tree.

\section{ACKNOWLEDGMENTS}

This work was supported by a scholarship (PhD - GDE) from CNPq (Brazil) and by Fundação Araucária/SETI (Brazil) under the project 45112, grant 144/15.

\section{REFERENCES}

[1] R. Baldoni, L. Querzoni, and A. Virgillito, "Distributed event routing in publish/subscribe communication systems: a survey," Tech. Rep., 2005.

[2] C. Esposito, D. Cotroneo, and S. Russo, "On reliability in publish/subscribe services," Comput. Netw., vol. 57, no. 5, pp. 1318-1343, Apr. 2013.

[3] M. Castro, P. Druschel, A. M. Kermarrec, and A. I. T. Rowstron, "Scribe: a large-scale and decentralized application-level multicast infrastructure," IEEE J. Sel. Areas Com., vol. 20, no. 8, pp. 1489-1499, Oct 2002.

[4] S. Q. Zhuang, B. Y. Zhao, A. D. Joseph, R. H. Katz, and J. D. Kubiatowicz, "Bayeux: An architecture for scalable and fault-tolerant wide-area data dissemination," in NOSSDAV '01, 2001, pp. 11-20.

[5] J. Gascon-Samson, F. Garcia, B. Kemme, and J. Kienzle, "Dynamoth: A scalable pub/sub middleware for latency-constrained applications in the cloud," in ICDCS, 2015, pp. 486-496.

[6] Y. Zhao, K. Kim, and N. Venkatasubramanian, "Dynatops: A dynamic topic-based publish/subscribe architecture," in DEBS, 2013, pp. 75-86.

[7] G. Cugola, E. Di Nitto, and A. Fuggetta, "The jedi event-based infrastructure and its application to the development of the opss wfms," IEEE Trans. Softw. Eng., vol. 27, no. 9, pp. 827-850, Sep. 2001.

[8] S. Bianchi, P. Felber, and M. G. Potop-Butucaru, "Stabilizing distributed r-trees for peer-to-peer content routing," IEEE Trans. Parallel Distrib. Syst., vol. 21, no. 8, pp. 1175-1187, 2010.

[9] P. T. Eugster, P. A. Felber, R. Guerraoui, and A.-M. Kermarrec, "The many faces of publish/subscribe," ACM Comput. Surv., vol. 35, no. 2, pp. 114-131, Jun. 2003.

[10] E. P. Duarte, Jr., L. C. E. Bona, and V. K. Ruoso, "VCube: A provably scalable distributed diagnosis algorithm," in Work. on Latest Advances in Scalable Algorithms for Large-Scale Systems, 2014, pp. 17-22.

[11] R. Prakash, M. Raynal, and M. Singhal, "An efficient causal ordering algorithm for mobile computing environments," in ICDCS, 1996, pp. $744-751$.

[12] S. Gao, G. Li, and P. Zhao, "Marshmallow: A content-based publishsubscribe system over structured $\mathrm{p} 2 \mathrm{p}$ networks," in 7th Int1l Conf. Comput. Intellig. Security, Dec 2011, pp. 290-294.

[13] A. Montresor and M. Jelasity, "Peersim: A scalable p2p simulator," in 2009 IEEE Ninth International Conference on Peer-to-Peer Computing, Sept 2009, pp. 99-100.

[14] J. P. de Araujo, L. Arantes, E. P. Duarte Jr., L. A. Rodrigues, and P. Sens, "A publish/subscribe system using causal broadcast over dynamically built spanning trees," 2017, preprint. [Online]. Available: https://arxiv.org/abs/1706.08302

[15] J. F. Kurose and K. W. Ross, Computer Networking: A Top-Down Approach (6th Edition), 6th ed. Pearson, 2012.

[16] R. Ramaswamy, N. Weng, and T. Wolf, "Characterizing network processing delay," in GLOBECOM, vol. 3, Nov 2004, pp. 1629-1634 Vol.3.

[17] C. Sanli and R. Lambiotte, "Local variation of hashtag spike trains and popularity in twitter," PLOS ONE, vol. 10, no. 7, pp. 1-18, 072015.

[18] E. Anceaume, R. Ludinard, A. Ravoaja, and F. Brasileiro, "Peercube: A hypercube-based p2p overlay robust against collusion and churn," in SSS, 2008, pp. 15-24.

[19] Y. Choi, K. Park, and D. Park, "Homed: a peer-to-peer overlay architecture for large-scale content-based publish/subscribe system," in DEBS, 2004, pp. 20-25.

[20] R. Baldoni, S. Bonomi, M. Platania, and L. Querzoni, "Dynamic message ordering for topic-based publish/subscribe systems," in IPDPS, 2012, pp. 909-920.

[21] C. Lumezanu, N. Spring, and B. Bhattacharjee, "Decentralized message ordering for publish/subscribe systems," in Middleware, 2006.

[22] K. Zhang, V. Muthusamy, and H. Jacobsen, "Total order in content-based publish/subscribe systems," in ICDCS, 2012, pp. 335-344. 